\title{
MICROCANTILEVERS FOR ATOMIC FORCE MICROSCOPE DATA STORAGE
}




\title{
MICROSYSTEMS
}

\author{
Series Editor \\ Stephen D. Senturia \\ Massachusetts Institute of Technology
}

\section{Editorial Board}

Roger T. Howe, University of California, Berkeley

D. Jed Harrison, University of Alberta

Hiroyuki Fujita, University of Tokyo

Jan-Ake Schweitz, Uppsala University 


\section{MICROCANTILEVERS FOR \\ ATOMIC FORCE MICROSCOPE \\ DATA STORAGE}

by

Benjamin W. Chui

Stanford University

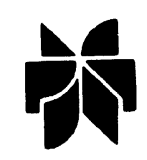

Springer Science+Business Media, LLC 
ISBN 978-1-4613-7262-2 ISBN 978-1-4615-4983-3 (eBook)

DOI 10.1007/978-1-4615-4983-3

\section{Library of Congress Cataloging-in-Publication Data}

A C.I.P. Catalogue record for this book is available from the Library of Congress.

Copyright (C) 1999 by Springer Science+Business Media New York Originally published by Kluwer Academic Publishers, New York in 1999 Softcover reprint of the hardcover 1st edition 1999

All rights reserved. No part of this publication may be reproduced, stored in a retrieval system or transmitted in any form or by any means, mechanical, photocopying, recording, or otherwise, without the prior written permission of the publisher, Springer Science+Business Media, LLC.

Printed on acid-free paper. 


\section{Editor's Preface}

This is the second volume in the new Microsystems Series from Kluwer Academic Publishers. Our Editorial Board has the goal of bringing to the Microsystems community, through rapid and timely publication, an outstanding library of reference works, monographs, textbooks, and selected Ph.D. dissertations.

The Microsystems field has an enormously broad scope, combining aspects of conventional microelectronics with non-electronic functionality in such diverse arenas as mechanical sensors, actuators, displays, vacuum microelectronics, chemical sensors and analysis systems, optical components, microfluidic components and systems, mass data storage, printing, switches and relays, even apparatus for research in cell biology and medicine. As our Series grows, it will include works from all these areas.

The first volume in this series (B. Romanowicz) dealt with Computer-Aided Design and modeling of Microsystem components. This second volume addresses the future of mass data-storage technologies. While progress in the speed and density of magnetic storage media continues to make larger mass memories available at successively lower costs, the question remains: What is next? In harnessing the techniques of scanning probe microscopy, specifically atomic force microscopy (AFM) in this case, Dr. Benjamin Chui has demonstrated, in his recent doctoral thesis at Stanford University (supervised by Prof. Thomas Kenny), that with suitable design, a single AFM tip can be used to write data thermally and also to read that data through 
piezoresistive sensing of the cantilever deflection. By pushing the state of the art, and then documenting in this volume the design,modeling, fabrication, and testing of prototype devices, Dr. Chui has both opened up a new technological area, and provided a case study that will serve both students and professionals in the Microsystems field.

Publication of outstanding books requires effort from many individuals-the author first and foremost, but also reviewers and publishers. On behalf of myself and our Editorial Board, our thanks to all concerned.

Stephen D. Senturia

Cambridge, MA 


\section{Foreword}

The density, speed and cost of mass data storage systems have improved steadily in recent years. In order to continue this trend, alternate technologies for data storage need to be evaluated and incorporated as appropriate. IBM is a world leader in data storage technology, and has also been a leading source of advanced technology. An excellent example of this technological leadership is the invention of the Scanning Tunneling Microscope at IBM Zurich.

This Ph.D. thesis describes a research collaboration between Mamin and Rugar's group at IBM Almaden and our group at Stanford University, in which a new mass data storage technology was evaluated. This technology is based on the use of heated cantilevers to form submicron indentations on a polycarbonate surface, and piezoresistive cantilevers to read those indentations.

There is a strong interdisciplinary component to this work. AFM and related tipbased interactions are generally in the domain of Applied Physics. The writing technology described here is dominated by heat transfer considerations. The dual-axis force sensor represents an effort in mechanical design and testing. The Ph.D. was awarded in Electrical Engineering, but this work is typical of the interdisciplinary nature of the MicroElectroMechanical Systems (MEMS) field.

The research described in this thesis was strongly enhanced by a close collaboration with Mamin and Rugar's IBM Almaden group; this collaboration provided access to 
testing facilities, contact with an appropriate industrial partner, and regular interactions with talented researchers. Collaborations with groups at Stanford University also provided support for microfabrication (Quate and Kovacs), assistance with measurements and analysis (Goodson), and many opportunities to exchange ideas and results.

Finally, Dr. Chui is the first Ph.D. student to complete his thesis under my supervision, and his work will always be memorable to me in that regard. Through this work, he has set a standard that subsequent students will struggle to duplicate, and he has helped build a reputation for our group that benefits us all.

Thomas W. Kenny

Stanford, CA 


\section{Abstract}

Atomic force microscope (AFM) thermomechanical data storage involves the recording of digital information as submicron data pits on a rotating substrate at very high densities up to $30 \mathrm{Gbit} / \mathrm{in}^{2}$. In a typical implementation, the sharp tip of a micromachined AFM cantilever is kept in continuous contact with a spinning polycarbonate disk by a weak loading force. For writing, the cantilever tip is heated above the glass transition temperature of polycarbonate to melt data pits onto the substrate. For reading, the cantilever deflection is measured as the tip rides over the pits. This work describes recent developments in AFM thermomechanical data storage, with a focus on the integration of heating and deflection-sensing elements onto the cantilevers.

Single-crystal silicon cantilevers $1 \mu \mathrm{m}$ thick have been demonstrated for use in AFM thermomechanical data storage. Cantilevers with integrated piezoresistive sensors were fabricated with measured sensitivities $\Delta R / R$ up to $7.5 \times 10^{-7}$ per $\AA$, in close agreement with theoretical predictions. Separate cantilevers with integrated resistive heaters were fabricated using the same basic process. Electrical and thermal measurements on these heating devices produced results consistent with ANSYS simulations. Geometric variants of the cantilever were also tested in order to study the dependence of the thermal time constant on device parameters. Depending on the design, time constants as low as $1 \mu \mathrm{s}$ were achieved. A thermodynamic model was developed based on the cantilever's geometry and material properties, and the model was shown to predict device behavior accurately. A comprehensive understanding of cantilever 
functionality enabled us to optimize the cantilever for high-speed thermomechanical recording.

A novel dual-axis piezoresistive cantilever has also been developed for tracking in AFM data storage. The cantilever was fabricated using a new technique for making high-aspect-ratio sidewall-implanted microstructures. The device allows simultaneous, independent detection of vertical and lateral forces. The vertical force sensor of the cantilever can be used for data readback and loading force control, while the lateral force sensor can be used for data groove edge detection and tracking. The dual-axis cantilever consists of a flat, triangular probe with a sharp tip, connected to the support by four parallel high-aspect-ratio ribs. Piezoresistors are fabricated on the horizontal surface of the triangular probe and the vertical sidewalls of the ribs for orthogonal deflection sensing. A special oblique ion implant at approximately $45^{\circ}$ to the normal is used to form the piezoresistors. Groove tracking on a spinning disk has been demonstrated using a custom two-channel servo controller for load regulation and tracking. Lateral force microscopy measurements as well as microscale friction measurements have also been performed using the dual-axis cantilever. 


\section{Acknowledgments}

It has been a very enjoyable and rewarding three-and-a-half years for me. It seemed like only yesterday that I started my Ph.D. program at Stanford, and before I know it, here I am finishing up my thesis. I guess I'll have to first thank El Niño for keeping me indoors during all these rainy days, so that I had no choice but to start writing....

First I'd like to acknowledge various funding sources for this work, including an International Business Machines Corporation Graduate Cooperative Fellowship, the Defense Advanced Research Projects Agency (contract no. DABT63-95-C-0019), a Terman Fellowship, and the NSF CAREER Program (no. ECS-9502046).

This work was a collaboration between Stanford University and IBM Almaden Research Center, and I believe I have benefited tremendously from the synergy that stemmed from this type of academic-industrial collaboration. I have numerous people to thank, both at Stanford and at IBM.

My studies at Stanford would not have been successful without the guidance and attention of my thesis advisor, Prof. Tom Kenny. He was always accessible whenever I had questions to ask or simply wanted to chat, and his refreshing humor was always welcome, as my fellow group members can attest to. Tom gave me a lot of freedom to pursue my project the way I wanted to, yet at the same time managed to keep tabs on my progress and give the right advice at the right time. Tom also kindly accommodated my outrageous requests for conference travel more times than once-I think I 
owe him a "Ben's world tour" T-shirt for the ten cities, six countries and three continents that I visited during my research-related trips.

Of equally valuable support to my Ph.D. work was Dr. John Mamin, my mentor at IBM Almaden Research Center. He used a significant portion of his time to help me get up to speed on the test apparatus at IBM labs, and taught me various aspects of AFM theory and operation. He kindly allowed me to include some of his AFM writing and readback results in my thesis. He was always available to answer any questions I had despite my sometimes unannounced visits to his office; I certainly feel very fortunate to have had John for my mentor.

I would also like to thank the rest of the exploratory storage group at IBM-namely Dr. Dan Rugar, Dr. Bruce Terris and Dr. Bob Ried-for their support and assistance. Dan was manager of the group and helped me formulate the overall strategy for my project. Bruce helped with major technical aspects of my assignment, particularly with the tracking experiment setup and the polymer test-disk fabrication. Bob and I had many useful discussions about cantilever design and fabrication. Dr. Long-sheng Fan was also an important contributor to the project. Acknowledgments are also due to Dr. Koichi Wago, Dr. Nino Yannoni and Dr. Doug Thompson at IBM. I am also grateful to numerous researchers at IBM Zurich Research Laboratory-including Dr. Gerd Binnig, Dr. Peter Vettiger, and Dr. Juergen Brugger-for sharing their valuable technical expertise with me, and for hosting me during a very enjoyable site visit in Switzerland.

This project would not have occurred without Dr. Storrs Hoen. He was the person who introduced the Kenny group to IBM Almaden, catalyzing collaborative research between the two parties, eventually leading to my IBM fellowship in connection with the AFM data storage project. I am very much indebted to Storrs indeed for his continued support.

I would like to thank the rest of my academic committee at Stanford University. Prof. Greg Kovacs, my associate advisor, with his expertise and vigor, has been giving me inspiration for my MEMS research since my M.S. years. I owe you a beer, Greg. Prof. Ken Goodson provided me with valuable advice on thermal subjects, and Prof. Cal Quate helped me benefit significantly from his group's expertise in atomic force microscopy. Prof. Robert Dutton, committee chairman, also devoted significant time and effort to matters in connection with my oral examination and thesis review. 
My fellow students in the Kenny group have always been a source of support and friendship. I'd like to thank Tim Stowe in particular for teaching me all about clean room processing when I first started. Other students with whom I spent numerous enjoyable hours in the lab and elsewhere included Kevin Yasumura, Jonah Harley, Aaron Partridge, Cheng-Hsien Liu, Kurth Reynolds, Aaron Barzilai, John Grade, Eugene Chow, Tim Pfafman, Alissa Fitzgerald, Yiching Liang, and Josh Molho, just to mention a few.

The staff at Stanford's semiconductor clean room-the Stanford Nanofabrication Facility-have provided valuable assistance throughout these years. I am grateful to Gladys, April, Robin, Marnel, Chris, Margaret, Nancy, Bob, Karl, Keith, Len, Luke, Pat, Bill, Sharleen, Mary, John, Tony, Mario, Joe and others for their untiring efforts at keeping the clean room up and running. I'd like to thank Gladys in particular for her help on the special oblique ion implantation process, which formed a vital part of my thesis.

I would like to thank all those who have given me advice or technical help at various times: Prof. Jim Plummer, Prof. Krishna Saraswat, Prof. David Bloom, Prof. Tom Lee, Prof. Simon Wong, Dr. Jim McVittie, Dr. Mark McCord, as well as James Kim. I would like acknowledge the continued support of Prof. Fabian Pease, who was my M.S. advisor at Stanford, and Prof. Martin Hellman, my undergraduate advisor at Stanford. In particular, I am grateful to various former and current Quate research group members for sharing their AFM expertise with me: Steve Minne, Tom Soh, Scott Manalis, Kathryn Wilder, Marco Tortonese, Rob Barrett, and Tom Albrecht.

I would like to thank students in other groups who were my lab and clean room buddies: Y.K. Leung, Alvin Loke, Steve Kuehne, Justin Leung, Patrick Yue, Vivek Subramanian, and T. C. Yang. I had very useful discussions with members of the Goodson group, including Sungtaek Ju, Mehdi Asheghi, Katsuo Kurabayashi, and Bill King. I also got a lot of help from the Kovacs group: John Suh, Bart Kane, Erno Klaassen, Dave Borkholder, Rich Reay, Ken Honer, Nick Mourlass, Brian Eplett, and many more. I would like to thank Sungtaek Ju in particular for his tremendous help and meticulous attention in proofreading this manuscript.

I would like to thank the Irwin family for their wonderful friendship and kindness all these years. A special note of appreciation is due to Darren Irwin, my Stanford undergraduate freshman roommate who, coincidentally enough, is pursuing his own Ph.D. I would also like to thank Mr. and Mrs. Matsunaga of Kyoto for their warm hospitality during my six-month stay in Japan, and Robert Chang and George Gray of 
the Japan SCTI program. I also wish to recognize some of my high-school mentors, including Mr. S. K. Wong, Mr. H. C. Li, Mr. M. K. Lee, Mr. K. I. Fong, Mr. C. K. Ma, and Mr. C. C. Chau.

I would not have enjoyed these years as much as I did without the company of my various groups of friends, including the DBS group, the SFSC group, as well as the CU- and MCS-related groups. I had a lot of fun with all you folks eating out, watching movies, cooking barbeques, playing tennis, playing games, singing karaoke, going on hiking trips, etc.

Most importantly, I am most grateful for the love and support of my mom, my dad and my sister all these years. I would not have been able to accomplish so much without them. Finally, I would like to dedicate this thesis to the memory of my beloved grandmother.

B. W. Chui

Stanford, CA 


\section{Contents}

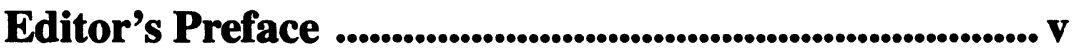

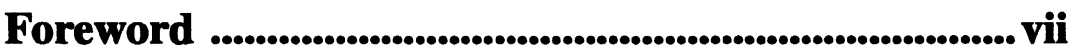

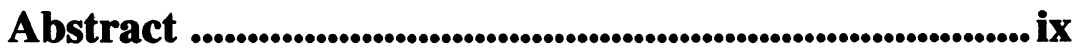

Acknowledgments ................................................................................ xi

Contents

List of Figures .......................................................................................xix

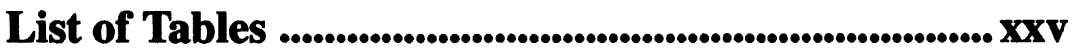




\section{Introduction} 1

1.1 High-density data storage: a survey ..........................................................1

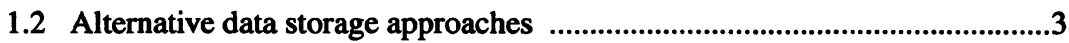

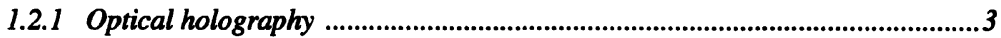

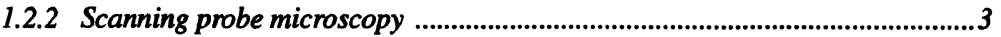

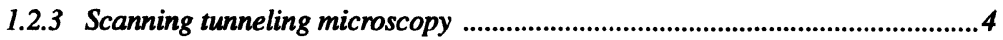

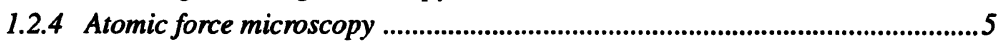

1.2.5 Near-field optics ....................................................................................6

1.3 AFM thermomechanical data storage ........................................................

\section{Heater-cantilevers for writing: design, fabrication} and basic characterization ...................................................... 11

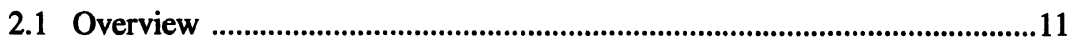

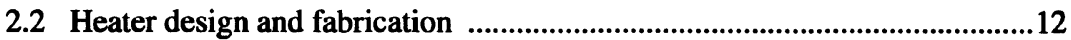

2.3 Thermal writing experiments ..............................................................16

2.4 Measuring temperature coefficients of resistance .......................................16

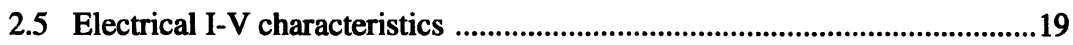

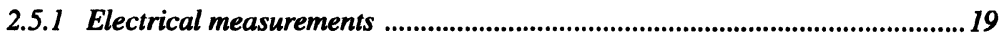

2.5.2 Thermal-electrical modeling .................................................................. 20

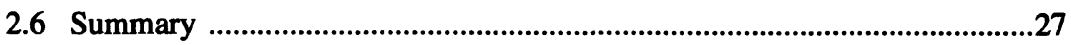

\section{Heater-cantilevers for writing: further character-} ization, modeling and optimization .................................. 29

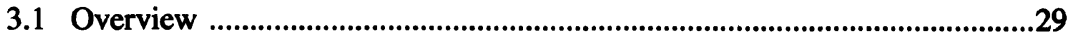

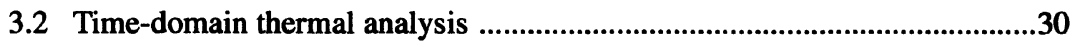

3.2.1 Thermal time constant: electrical measurements ............................................30

3.2.2 Finite element modeling ............................................................................33

3.2.3 Comparison of heat loss mechanisms from heater ........................................33

3.2.4 Thermal time constant: laser thermometry measurements .............................41

3.3 Frequency-domain thermal analysis ..........................................................42

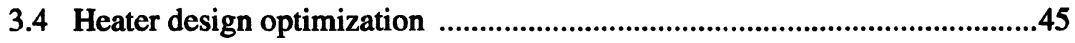

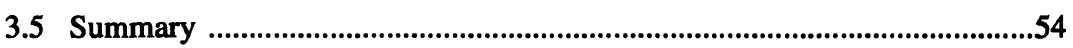


4 Piezoresistive cantilevers for readback .......................... 55

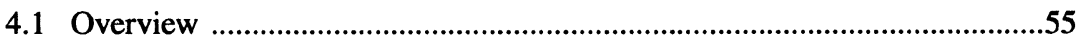

4.2 Piezoresistive cantilever design analysis ...................................................56

4.2.1 Mechanical stiffness requirement ...............................................................56

4.2.2 Piezoresistive sensitivity requirement ............................................................57

4.2.3 Resonant frequency considerations .............................................................60

4.2.4 Overall cantilever specifications .................................................................61

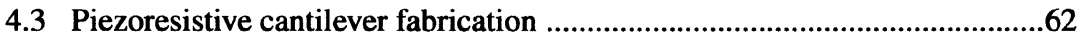

4.4 Characterization of piezoresistive cantilevers .........................................67

4.4.1 Piezoresistive sensitivity ..........................................................................67

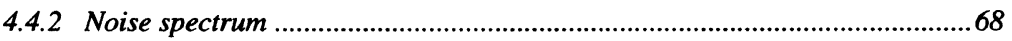

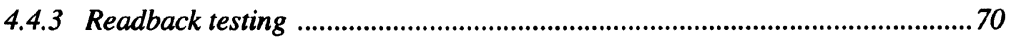

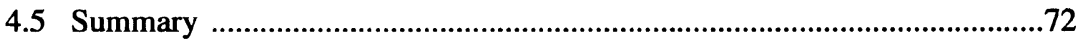

\section{Dual-axis piezoresistive cantilevers: design,} fabrication and characterization ...................................... 73

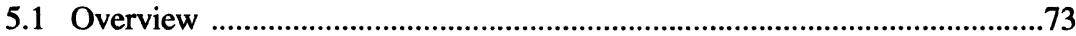

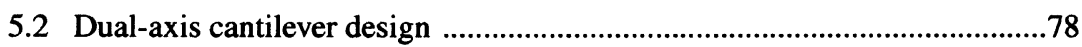

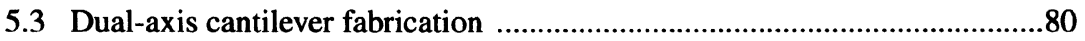

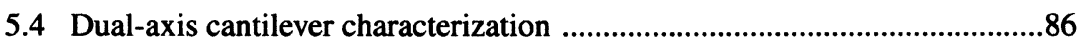

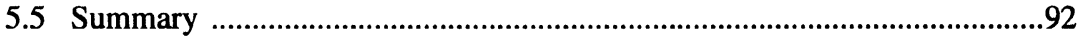

\section{Dual-axis piezoresistive cantilevers for tracking:} applications ............................................................................. 93

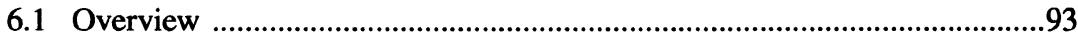

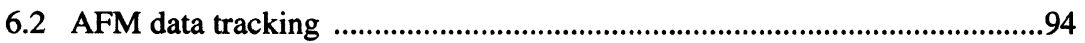

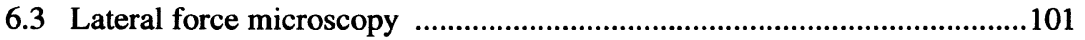

6.3.1 Dual-channel AFM imaging .......................................................................101

6.3.2 Microscale friction measurements .................................................................. 103

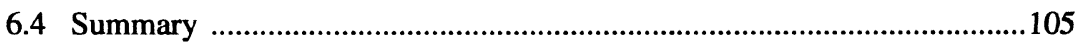


7 Conclusion and future work ......................................... 107

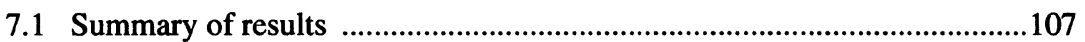

7.1.1 Low-stiffness piezoresistive cantilevers .....................................................107

7.1.2 Cantilevers with integrated resistive heaters ............................................... 108

7.1.3 Dual-axis piezoresistive cantilevers ………….............................................108

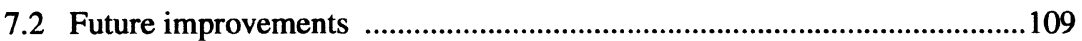

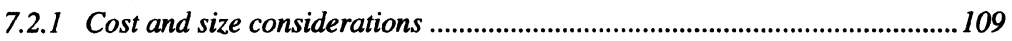

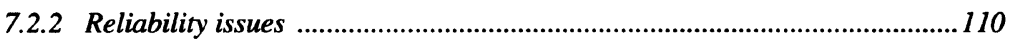

7.2.3 Data rate improvements .............................................................................111

7.2.4 Bit areal density improvements .................................................................114

7.2.5 Outlook for the future ..............................................................................115

Appendix 1 Heater-cantilever fabrication process .............................................................. 117

Appendix 2 Piezoresistive cantilever fabrication process ............................................................. 121

\section{Appendix 3 Dual-axis piezoresistive cantilever fabrication process ...................................... 125}

Bibliography

Index 


\section{List of Figures}

\section{Introduction}

FIG. 1-1 Areal density history of IBM's magnetic disk drives, showing a recent compound growth rate of $60 \%$

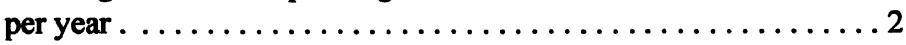

FIG. 1-2 Comparison of different approaches to data storage. $\ldots \ldots \ldots 6$

FIG. 1-3 Original configuration of thermomechanical data storage test setup using laser-based reading and writing. . . . . . . . 8

FIG. 1-4 Integrating piezoresistive sensors and resistive heaters on micromachined cantilevers for AFM thermomechanical data storage. . . . . . . . . . . . . . . . . 9 


\section{Heater-cantilevers for writing: design, fabrication and basic characterization}

FIG. 2-1 Schematic of cantilever with integrated resistive heater $\ldots \ldots \ldots 12$

FIG. 2-2 Fabrication process for $1-\mu \mathrm{m}$ thick heater cantilevers. . . . . . 13

FIG. 2-3 SEM images of (a) Type A, boron-doped, "non-constricted"

heater-cantilever, and (b) Type B, phosphorus-doped, "constricted" heater. Part (c) shows a perspective view of a Type $B$ heater-cantilever. . . . . . . . . . . . . . . 15

FIG. 2-4 AFM image of sample data tracks written with (a) Type A heater using $16 \mathrm{~V}, 20 \mu$ s pulses with a period of 90-200 $\mu \mathrm{s}$ (up to $11 \mathrm{kbit} / \mathrm{s}$ ); (b) Type B heater using $30 \mathrm{~V}, 0.2 \mu$ s pulses with a period of 7-14 $\mu$ s (up to $140 \mathrm{kbit} / \mathrm{s}$ ). Part (c) shows another thermal writing sample. ................ 17

FIG. 2-5 Resistance vs. temperature measurements for Type A heater (top) and Type B heater (bottom) $\ldots \ldots \ldots \ldots \ldots \ldots \ldots \ldots$

FIG. 2-6 Electrical I-V curves obtained (a) with a semiconductor parametric analyzer using a slow DC sweep from 0 to $10 \mathrm{~V}$, and (b) with short voltage pulses and a sense resistor and oscilloscope to measure instantaneous current...........

FIG. 2-7 Variation of intrinsic carrier concentration in silicon vs. temperature............................. 22

FIG. 2-8 Simple thermal-electrical model of heater-cantilever... . . . . . 23

FIG. 2-9 Room-temperature thermal conductivity data for silicon layers as a function of their thickness $\ldots \ldots \ldots \ldots \ldots \ldots \ldots \ldots 24$

FIG. 2-10 (a) Simulated I-V curve based on thermal runaway hypothesis compared with measured curve. (b) Estimated carrier concentration and temperature vs. applied voltage. (c) Estimated resistance of cantilever leg and heater region vs. voltage. .....26

FIG. 2-11 Optical photograph of glowing hot heater-cantilever, taken with the cantilever's own emitted light. ............ 27 


\section{Heater-cantilevers for writing: further character- ization, modeling and optimization}

FIG. 3-1 Electrical measurements for estimating Type A heater temperature during and after a heating pulse. ........... 31

FIG. 3-2 Time-domain temperature variation of Type B heater subjected to single heating pulse.

FIG. 3-3 Finite element models used to represent Type A heater (top) and Type B heater (bottom).

FIG. 3-4 Simple thermal model of heater-cantilever used to calculate relative rates of heat dissipation by radiation, convection and conduction.

FIG. 3-5 (a) Reflected laser power from Type A heater subjected to heating pulse, and (b) Laser vibrometer signal showing mechanical vibration of cantilever tip.

FIG. 3-6 (a) Frequency-dependent temperature fluctuation of Type B heater subjected to sinusoidally varying input power, and

(b) Capacitor-resistor thermodynamic model based on the cantilever's geometry and material properties.

FIG. 3-7 Measured and simulated thermal time constants for families of Type B heaters, under repeated-pulse conditions... . . . . 46

FIG. 3-8 Simple model illustrating one way in which the cantilever leg width affects the thermal time constant.

FIG. 3-9 Graphical representation of mathematical optimization of heater geometry.

FIG. 3-10 Theoretical prediction of optimal thermal time constant possible for given cantilever thicknesses.

FIG. 3-11 Predicted resonant frequency (thick solid line) vs. cantilever length for selected values of cantilever thickness........... 53 


\section{Piezoresistive cantilevers for readback}

FIG. 4-1 Combinations of cantilever thickness $d$ and length $l$ that satisfy mechanical stiffness requirements (solid lines) and piezoresistive sensitivity requirements (dotted lines)........ 57

FIG. 4-2 Piezoresistive coefficients in p-type silicon as a function of crystal orientation in the (001) plane at room temperature.. . . . . 58

FIG. 4-3 Piezoresistive factor vs. doping concentration in p-type silicon at $25^{\circ} \mathrm{C} . \ldots \ldots \ldots \ldots \ldots \ldots \ldots \ldots \ldots \ldots . \ldots \ldots$

FIG. 4-4 Side-view schematic of piezoresistive cantilever

FIG. 4-5 Combinations of cantilever thickness $d$ and length $l$ that satisfy mechanical stiffness requirements (solid lines) and resonant frequency requirements (dotted lines). ..........61

FIG. 4-6 Fabrication process for $1-\mu \mathrm{m}$ thick piezoresistive cantilevers. . . . 63

FIG. 4-7 Tip formation by undercutting resist-oxide cap (left) and further etching with the resist removed (right)...........64

FIG. 4-8 Spreading resistance profile (heavy line) showing a boron piezoresistive layer only $0.4 \mu \mathrm{m}$ in depth.............65

FIG. 4-9 SEM images of $75 \mu \mathrm{m}$ long cantilever and close-up view of sharp tip. .......................... 66

FIG. 4-10 Piezoresistive sensitivity vs. length for various cantilevers. . . . 68

FIG. 4-11 Noise spectrum of a $75-\mu$ m piezoresistive cantilever.. . . . . . 69

FIG. 4-12 Readback signal obtained with 75- $\mu$ m piezoresistive cantilever operated on spinning test sample with $6000 \AA$ deep grooves.. . . . 70

FIG. 4-13 Readback signals obtained simultaneously with two $150 \mu \mathrm{m}$ long piezoresistive cantilevers operated on a spinning test sample with a $1100 \AA$ deep features................ 71

FIG. 4-14 SEM image of four-cantilever array, fabricated in the same process as the individual cantilevers in this chapter......... 72 


\section{Dual-axis piezoresistive cantilevers: design, fabrication and characterization}

FIG. 5-1 Schematic showing how mis-centering of the data disk with respect to the spindle axis results in runout. ............ 74

FIG. 5-2 Principle of topographical AFM data tracking. . . . . . . . 75

FIG. 5-3 Schematic showing torsional bending mode (left) and vertical bending mode (right) of a simple planar cantilever. . . . . . 76

FIG. 5-4 Novel dual-axis AFM cantilever with orthogonal axes of compliance and independent piezoresistive sensors for vertical and lateral deflections. . . . . . . . . . . . . 78

FIG. 5-5 Vertical and lateral bending modes of dual-axis cantilever. . . . . 79

FIG. 5-6 Fabrication process for dual-axis cantilever............81

FIG. 5-7 Tip formation process as a function of time. . . . . . . . 82

FIG. 5-8 Thick photoresist used to mask ribs or other tall features during oblique ion implantation. . . . . . . . . . . . 84

FIG. 5-9 SEM image of dual-axis cantilever $\ldots \ldots \ldots \ldots \ldots \ldots$

FIG. 5-10 Noise spectra of vertical and lateral piezoresistive deflection sensors............................ 87

FIG. 5-11 Electrical crosstalk between the vertical and lateral deflection sensors as a function of frequency. .............. 88

FIG. 5-12 Synchronous signals from the vertical and lateral deflection sensors on a dual-axis cantilever as it is scanned from side to side with the tip in contact with a polycarbonate substrate.

FIG. 5-13 Piezoresistive deflection sensor signals from cantilever after being subjected to mechanical impulse, showing resonant behavior in vertical direction and lateral direction..........90

FIG. 5-14 ANSYS finite element model used to predict natural frequencies of the dual-axis cantilever structure. . . . . . . 91 


\section{Dual-axis piezoresistive cantilevers for tracking: applications}

FIG. 6-1 Schematic showing dual-axis cantilever mounted on compact disk actuator for AFM data tracking................. 95

FIG. 6-2 Experimental setup for tracking tests with dual-axis cantilever... 96

FIG. 6-3 Block diagram of one channel of the servo system used in tracking experiments........................ 97

FIG. 6-4 Circuit schematic for servo controller. . . . . . . . . . . . . . 97

FIG. 6-5 Closed-loop frequency response of track servo. .......... 98

FIG. 6-6 Dark-field optical photograph of silicon master for "data tracks".................................99

FIG. 6-7 Servo output signals and lateral error signals measured during groove-tracking test on spinning disk, with (a) lateral track servo off, and (b) lateral track servo on.

FIG. 6-8 Vertical deflection signal from the dual-axis cantilever while tracking an intermittent groove that emulates a "101010...." data pattern. ............................... 101

FIG. 6-9 (a) Vertical force AFM image and (b) lateral force AFM image taken in parallel during one scan with a dual-axis cantilever.

(c),(d) Close-up AFM images of a single ridge in each direction, obtained on the same sample. ..................... 102

FIG. 6-10 Line scans across a ridge on the sample of Figure 6-9....... 104

FIG. 6-11 Measured lateral force vs. applied loading force for silicon tip scanned across polycarbonate surface in air at various tip velocities.

\section{Conclusion and future work}

FIG. 7-1 SEM image of a $0.34 \mu \mathrm{m}$ thick, $12 \mu \mathrm{m}$ long, $4.6 \mathrm{MHz}$ silicon piezoresistive cantilever with an INCISIVE tip........... 112 


\section{List of Tables}

\section{Heater-cantilevers for writing: further character- ization, modeling and optimization}

TABLE 3-1 ANSYS finite element analysis parameters. Note the use of temperature-dependent material properties (thermal conductivity, specific heat, and electrical resistivity)......... 36

TABLE 3-2 Comparison of heat loss mechanisms from heater element ....... 39

\section{Piezoresistive cantilevers for readback}

TABLE 4-1 Comparison of piezoresistive cantilevers from this work, cantilevers from Tortonese, et al., and commercial devices from Park Scientific Instruments. . . . . . . . . . . . . . 67 